\title{
Tetramethypyrazine inhibits renal cell carcinoma cells through inhibition of NKG2D signaling pathways
}

\author{
YUN LUAN $^{1 *}$, JUNLI LIU ${ }^{2 *}$, XIAOLI LIU ${ }^{3}$, XIA XUE $^{4}$, FENG KONG $^{1}$, CHAO SUN $^{1}$, \\ JUE WANG $^{1}$, LING LIU ${ }^{2}$ and HONGYING JIA ${ }^{5}$ \\ ${ }^{1}$ Central Research Laboratory, ${ }^{2}$ Clinical Molecular Biology Laboratory, ${ }^{3}$ Department of Hematology, \\ ${ }^{4}$ Department of Pharmacy, ${ }^{5}$ Center of Evidence-Based Medicine, The Second Hospital \\ of Shandong University, Jinan, Shandong 250033, P.R. China
}

Received June 2, 2016; Accepted August 2, 2016

DOI: $10.3892 /$ ijo.2016.3670

\begin{abstract}
Tetramethypyrazine (TMP), one of the active compounds extracted from the traditional Chinese medicinal herb(Chuanxiong), has been verified as an anticancercompound against several types of cancer. However, understanding of the molecular mechanisms have not been fully elucidated. In the present study, the anticancer efficacy of TMP was investigated in human clear cell renal cell carcinoma (ccRCC) cells. We showed that TMP significantly inhibited ccRCC cell viability, proliferation, apoptosis, invasion and migration through the methods of MTT, flow cytometry, wound healing and Transwell assays. Furthermore, reverse transcription polymerase chain reaction (RT-PCR), western blotting and immunofluorescence results demonstrated TMP upregulation of the expression of NKG2D ligands (NKG2DLs) MHC class I chain-related molecules A and B (MICA/B) and epithelial cell expression marker of E-cadherin, and downregulation of mesenchymal cell expression markers of vimentin and fibronectin. Taken together, the inhibition of TMP on ccRCC cells might be mediated via inhibition of NKG2D related signaling pathway to further suppress epithelial-mesenchymal transition (EMT) progression. The binding of NKG2D to its ligands activates NK cells, giving the rationale for studies on the utilization of TMP as a potential cancer therapeutic compound to increase NK cells-mediated cytotoxicity against high MICA/B expression in cancer cells.
\end{abstract}

\section{Introduction}

2,3,5,6-tetramethylpyrazine (TMP) is an active alkaloid extracted from the traditional Chinese medicinal herb

Correspondence to: Professor Hongying Jia, Center of EvidenceBased Medicine, The Second Hospital of Shandong University, 247\#, Beiyuan Dajie, Jinan, Shandong 250033, P.R. China

E-mail: jiahongying_sd@163.com; jiahongying@sdu.edu.cn

*Contributed equally

Key words: 2,3,5,6-tetramethylpyrazine, NKG2D, MICA/B, clear cell renal cell carcinoma, epithelial-mesenchymal transition
Ligusticum chuanxiong, which has been widely used in the clinical treatment of neurovascular, cardiovascular and liver diseases in China (1-6). In recent years, increasing number of studies have suggested that TMP possesses anticancer properties (7-11). However, the mechanisms have yet to be elucidated. Epithelial-mesenchymal transition (EMT) is a developmental process characterized by the acquisition of invasiveness in epithelial tumors frequently accompanied by loss of their polarity and acquiring migratory properties of mesenchymal cells (12), which has been viewed as a critical process of invasion and metastasis in various types of cancer cells (13) associated with drug resistance (14).

Natural killer (NK) cells are a type of cytotoxic lymphocyte capable of mediating early innate immune responses to viral infections and recognition of transformed malignant cells $(15,16)$. $\mathrm{NK}$ group 2, member $\mathrm{D}(\mathrm{NKG} 2 \mathrm{D})$ is an activating receptor expressed by $\mathrm{NK}$ and $\mathrm{T}$ cells that play a major role in cancer immunosurveillance (17). MHC class I chain-related molecules A and B (MICA/B) and UL16 binding protein (ULBP) 1-6 molecules are the ligands of NKG2D (NKG2DLs), upregulation of MICA/B and ULBP in tumor cells suggests that NKG2D may exerts a key role in response to infection and transformation $(18,19)$. However, the regulation of the NKG2D signaling during the EMT of carcinomas have yet to be elucidated.

Clear cell renal cell carcinoma (ccRCC) is one of the most common kidney cancers, and there are several studies showing that EMT is associated with carcinoma invasion and metastasis, but the relationship between ccRCC and EMT is not yet clarified (20). In our previous study (21), we found that the content of soluble MICA (sMICA) in serum of patients with renal cell carcinoma was significantly higher than in healthy adults, the percentage of membrane MICA (mMICA) were significantly increased in human kidney cancer tissues and ccRCC cell lines. Furthermore, we indicated that the relationship of expression of NKG2D and MICA/B play an important role in mediation of the cytotoxic NK cells in ccRCCs and sMICA-mediated tumor immune escape through downregulated NKG2D expression. In the present study, we tested the hypothesis that TMP inhibits proliferation, migration and invasion of ccRCC cells through regulation of NKG2D-MICA signaling during the EMT by using the ccRCC cell lines 786-O and A498. 


\section{Materials and methods}

Drugs and cell culture. Tetramethypyrazine (TMP Enterprises, St. Louis, MO, USA) was dissolved in dimethyl sulfoxide (DMSO; Sinopharm Chemical Reagent Co., Ltd., Shanghai, China) as a stock solution. The dilutions of all of the reagents were freshly prepared before each experiment. Hence, normal saline was used as a vehicle control in all experiments. RCC cell lines (786-O, A498) were obtained from the Chinese Academy of Medical Sciences and maintained in RPMI-1640 (HyClone Laboratories, Inc., Logan, UT, USA) supplemented with $10 \%(\mathrm{v} / \mathrm{v})$ heat-inactivated fetal bovine serum (FBS; Gibco, Carlsbad, CA, USA), containing $100 \mathrm{U} / \mathrm{ml}$ penicillin and $100 \mu \mathrm{g} / \mathrm{ml}$ streptomycin in a humidified atmosphere of $5 \% \mathrm{CO}_{2}$ at $37^{\circ} \mathrm{C}$. Cells were harvested by brief incubation in $0.02 \%(\mathrm{w} / \mathrm{v})$ EDTA in PBS. In blocking experiments, antiMICA/B mAbs were added to the RCC cell suspension at $10 \mu \mathrm{g} / \mathrm{ml}$ and incubated at $37^{\circ} \mathrm{C}$ for $30 \mathrm{~min}$ before the addition of target cells.

Growth and cell viability analysis. The viability of ccRCC cells was evaluated by 3-(4,5-dimethylthiazol-2-yl)-2, 5-diphenyltetrazolium bromide (MTT) assay. Briefly, cells $\left(5 \times 10^{3} /\right.$ well) seeded in 96-well plates were incubated with increasing concentrations of TMP for 24,48 and $72 \mathrm{~h}$, respectively. The controls were treated with an equal volume of the drug vehicle DMSO, but the applied concentration did not exhibit a modulating effect on cell growth. Optical density (OD) of each culture was read by Universal Microplate Reader at wavelength of $570 \mathrm{~nm}$.

Reverse transcription polymerase chain reaction (RT-PCR). Total RNA was extracted from kidney tissue using a Qiagen RNeasy kit (Qiagen, Basel, Switzerland). Complementary DNA (cDNA) First Strand was produced using a SuperScript First-Strand Synthesis system using oligo (dt) antisense primers (Invitrogen, Lucerne, Switzerland). MICA and MICB transcripts were amplified from cDNA by 30 cycles of polymerase chain reaction. The primer sequences were as follows: MICA (517 bp) upstream (5'-ACACCCAGCAGTGGGGG GAT-3'); downstream primer (5'-GCAGGGAATTGAATCCC AGCT-3'); MICB (678 bp) upstream '5-ACACCCAGCAGT GGGGGGAT-3', downstream 5'-AGCAGTCGTGAGTTTGC CCAC-3'. The amplification conditions consisted of denaturizing $\left(95^{\circ} \mathrm{C}\right.$ for $\left.60 \mathrm{sec}\right)$, followed by annealing $\left(56^{\circ} \mathrm{C}\right.$ for $\left.60 \mathrm{sec}\right)$, and extension $\left(72^{\circ} \mathrm{C}\right.$ for $\left.60 \mathrm{sec}\right)$. Amplified fragments were analyzed in $1.5 \%$ agarose gel electrophoresis in the presence of ethidium bromide (Sigma-Aldrich). $\beta$-actin (500 bp) was used as an internal control for the amount of RNA input.

Flow cytometry. The Annexin V-FITC/PI apoptosis detection kit according to the manufacturer's instructions (Roche Diagnostics, Indianapolis, IN, USA). Briefly, ccRCC cells were seeded in 6-well plates and treated with $100 \mu \mathrm{M} \mathrm{TMP}$ for $48 \mathrm{~h}$. Cells $1 \times 10^{6}$ were collected and suspended in $500 \mu \mathrm{l}$ binding buffer, and $5 \mu \mathrm{l}$ Annexin V-FITC and $5 \mu \mathrm{l}$ PI were added to each sample and incubated in the dark for $15 \mathrm{~min}$. The cell surface phosphatidylserine in apoptotic cells was quantitatively estimated by using Annexin V/FITC apoptosis detection kit according to manufacturer's instructions (Roche Diagnostics).
Data were detected using FACScan flow cytometry (FACS LSRFortessa; BD Biosciences, San Jose, CA, USA). Triplicate experiments with triplicate samples were performed.

Terminal deoxynucleotidyl transferase-mediated deoxyuridine triphosphate nick-end labelled. Apoptosis was detected using a One Step TUNEL apoptosis assay kit (Beyotime Institute of Biotechnology, Shanghai, China) according to the protocol. Briefly, $5 \times 10^{3}$ cells were seeded on coverslips and cultured in RPMI-1640 with or without $100 \mu \mathrm{M}$ TMP containing $10 \%$ FBS in 24-well plates for $24 \mathrm{~h}$. After fixing with $4 \%$ formaldehyde, the cells were washed with PBS and permeabilized using $0.2 \%$ Triton X-100. Following equilibration, cells were labeled using TdT reaction mix and incubated for $1 \mathrm{~h}$ at $37^{\circ} \mathrm{C}$ in a humidified chamber. Subsequently, 2X SSC was added for $15 \mathrm{~min}$ to stop the reaction. Apoptotic cells were detected using a Nikon Eclipse 90i microscope (Nikon Corp., Tokyo, Japan) (22).

Wound healing assay. Cells $\left(5 \times 10^{5}\right)$ were plated onto 24 -well plates per well and were grown to $>90 \%$ confluence for $48 \mathrm{~h}$. The medium was removed, and in the cell monolayers a wound line was created by manually scraping the cells with a $10 \mu \mathrm{l}$ plastic pipette tip. Debris was removed from the culture by washing with PBS two times, and then the cells cultured in RPMI-1640 containing 1\% FBS with TMP (at $100 \mu \mathrm{M}$ ). Images (magnification, $\mathrm{x} 40$ ) were taken at 0 and $24 \mathrm{~h}$ using a Nikon Eclipse 90i microscope (Nikon Corp.). These experiments were repeated three times.

Transwell migration and invasion assays. The invasion of RCC cells were determined using a Transwell assay, briefly, experiments were performed in RPMI-1640 containing 10\% FBS as a chemoattractant. Following $100 \mu \mathrm{M}$ TMP treatment for $48 \mathrm{~h}$, cells were re-suspended in RPMI-1640 containing $1 \% \mathrm{FBS}$ and added $\left(1 \times 10^{6}\right.$ cells $\left./ 100 \mu \mathrm{l}\right)$ to the upper chamber consisting of a polycarbonate membrane $(8-\mu \mathrm{m}$ pore size; Corning Life Science). After a 48 -h incubation at $37^{\circ} \mathrm{C}$ under $5 \% \mathrm{CO}_{2}$, cells that had not migrated were removed, whereas migrated cells were fixed in $4 \%$ paraformaldehyde for $10 \mathrm{~min}$ at room temperature and stained with Crystal Violet Staining Solution kit (Beijing Solarbio Science and Technology Co., Ltd., Beijing, China). The number of migrated cells were counted using Nikon Eclipse 90i microscope.

Western blotting. Western blotting was performed to analyze the expression of apoptotic and EMT signal pathway-related proteins in ccRCC cells. Briefly, 786-O and A498 cells seeded in 6-well plates were exposed to $100 \mu \mathrm{M}$ TMP for $48 \mathrm{~h}$. The cells were harvested and lysates were fractionated by $10 \%$ SDS-PAGE. The proteins were electro-transferred onto PVDF membranes, and then the membranes were blocked in 5\% skimmed milk-Tris-buffered saline plus Tween-20 solution and incubated with primary antibodies E-cadherin, vimentin, fibronectin, matrix metalloproteinase-9 (MMP-9) and TGF- $\beta 1$. Following incubation with peroxidase-conjugated AffiniPure goat anti-rabbit IgG (1:5,000; ZSGB-Bio Co., Ltd., Beijing, China). The bound antibodies were visualized by using an enhanced chemiluminescence reagent (Millipore, Billerica, MA, USA) followed by Bio-Rad Image 
$\mathrm{Lab}^{\mathrm{TM}}$. Data was expressed as the relative density of the protein normalized to $\beta$-actin. The percentages of increase or decrease of protein were estimated by comparison to vehicle control (100\%).

Immunofluorescence staining analysis. In order to investigate the inhibition mechanism of TMP on ccRCC cell line, the epithelial-mesenchymal transition signal pathway-related markers E-cadherin, vimentin and fibronectin were detected by cell immunofluorescence techniques. Cells were seeded on coverslips in 6 -well plates $\left(6 \times 10^{4} /\right.$ well $)$ with or without treated with $100 \mu \mathrm{M}$ TMP for $24 \mathrm{~h}$, and then fixed in $4 \%$ paraformaldehyde for $30 \mathrm{~min}$ and washed three times with PBS. The slips were permeabilized for $10 \mathrm{~min}$ with $0.1 \%$ Triton X-100 and washed three times with PBS, and then cells were blocked with $10 \%$ normal goat serum for $30 \mathrm{~min}$. Cells were incubated overnight at $4^{\circ} \mathrm{C}$ with primary antibodies against E-cadherin (1:100 dilution; Cell Signaling Technology), vimentin (1:100, ab92547; Abcam). Later they were stained in the dark with tetraethyl rhodamine isothiocyanate (TRITC) (1:200; ZSGB-Bio Co.) as secondary antibodies. Images were taken with a Nikon Eclipse 90i microscope. The staining was analyzed with the image-analyzing system, Image-Pro Plus 6 (Media Cybernetics, Inc., Rockville, MD, USA).

Isolation of natural killer cells. Primary human NK cells were were isolated with an NK cell isolation kit (Miltenyi Biotec,Inc., Auburn, CA, USA) and obtained as previously described (23). Briefly, peripheral blood lymphocytes were isolated by density gradient centrifugation (Biochrom $\mathrm{GmbH}$, Berlin, Germany) from heparinized venous blood obtained from normal healthy volunteer donors and depletion of plastic-adherent monocytic cells. Isolation of highly pure NK cells was achieved by depletion of magnetically labeled cells. The percentage of NK cells after isolation was evaluated using FITC-conjugated anti-CD3 mAbs and PE-conjugated anti-CD56 mAbs (BD Biosciences) in flow cytometry, and routinely exceeded $95 \%$, with CD3 contamination in purified NK cells typically $<1 \%$.

Cytotoxicity assay. NK cell cytotoxicity against renal carcinoma cell lines was assessed using the calcein-AM release assay, as previously described (24). Renal carcinoma target cells (T) were cultured in medium with or without $100 \mu \mathrm{M}$ TMP for $48 \mathrm{~h}$. Then, the NK cell cytotoxicity assay was carried out by incubating these cells with NK effector cells (E) at various $\mathrm{T} / \mathrm{E}$ ratios $(1 / 20-1 / 5)$ for $3 \mathrm{~h}$ at $37^{\circ} \mathrm{C}$. In blocking experiments, anti-MICA/B mAbs were added to the NK cell suspension and incubated at $37^{\circ} \mathrm{C}$ for $30 \mathrm{~min}$ before the addition of target cells. The number of dead cells was estimated by measuring the fluorescence intensity in medium. The fluorescence intensity in medium released spontaneously from target cells and the total maximum fluorescence intensity released from all target cells by treatment with $1 \%$ Triton X-100 were also determined.

Statistical analysis. Data of continuous variables are presented as means $\pm \mathrm{SD}$. Comparisons between the treatments were analyzed by one-way ANOVA and performed with SPSS 10.0 software. $\mathrm{P}<0.05$ was deemed statistically significant.

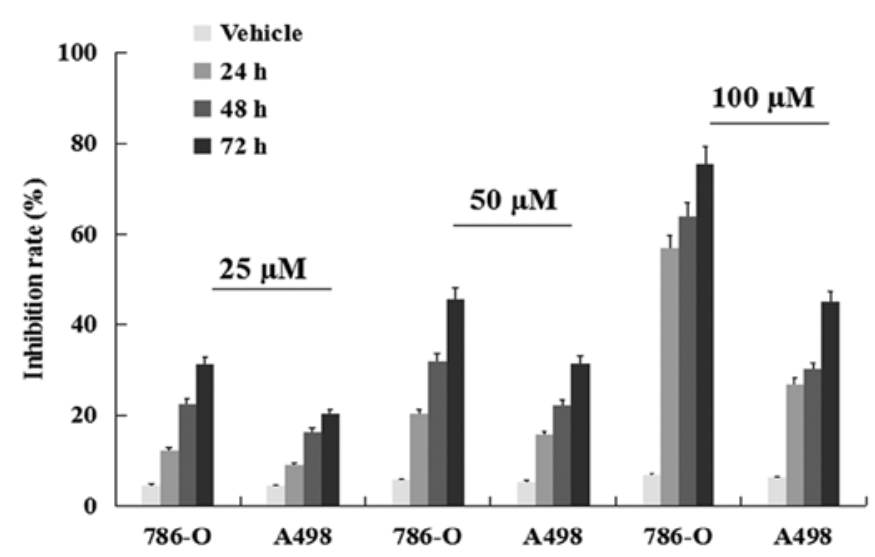

Figure 1. The growth inhibition effect of ccRCC cells induced by TMP in MTT assay. ccRCC cell lines were treated with TMP for 24, 48 and $72 \mathrm{~h}$, respectively.

\section{Results}

Inhibition of viability. ccRCC cell lines were treated with TMP for 24,48 and $72 \mathrm{~h}$, respectively and then subjected to the MTT assay. TMP effectively inhibited the viability of human clear cell renal cell carcinoma 786-O and A489 cells. As shown in Fig. 1, the inhibition rate increased from 24 to $72 \mathrm{~h}$. The maximum inhibition rate of $75.63 \%$ was found with use of $100 \mu \mathrm{M}$ for $72-\mathrm{h}$ treatment in 786-O. These results indicated that TMP had a dose- and time-dependent antiproliferative effect on ccRCC cells in the range of 25-100 $\mu \mathrm{M}$ for up to 24 , 48 and $72 \mathrm{~h}$ of exposure. Moreover, a better inhibition rate was shown in $786-\mathrm{O}$ than $\mathrm{A} 498$ cell line $(\mathrm{P}<0.05)$.

TMP induction of ccRCC cell apoptosis. To evaluate the TMP-induced cell apoptosis, ccRCC cells were then stained with Annexin V/FITC and were analyzed by flow cytometry. As shown in Fig. 2, the percentage of apoptotic cells was gradually increased in 786-O and A498 after treatment $24 \mathrm{~h}$ with TMP at the concentrations of 25,50 and $100 \mu \mathrm{M}$. Furthermore, we examined the morphologic changes in 786-O by TUNEL staining as shown in Fig. 3. When cells were cultured with $100 \mu \mathrm{M}$ TMP for $24 \mathrm{~h}$, the apoptotic morphologic changes were observed as compared with the vehicle control.

Inhibition of cell migration and invasion. To determine whether TMP has effects on the migration and invasion of ccRCC cells, the wound healing and Transwell assays were performed. As demonstrated in Figs. 4 and 5, the migratory ability of the 786-O was significantly inhibited after treatment of the cells with $100 \mu \mathrm{M}$ of TMP at 6 and $12 \mathrm{~h}$ through wound healing assay. On the other hand, in the concentrations of 25 , 50 and $100 \mu \mathrm{M}$ TMP clearly inhibited migration and invasion ability of cells through a Transwell insert filter at $24 \mathrm{~h}$. Together, these results demonstrated that TMP inhibition of migration and invasion of ccRCC cells was dose- and timedependent.

Inhibition of epithelial-to-mesenchymal transition markers, $M M P-9$ and TGF- $\beta 1$. Immunofluorescence techniques and 
A

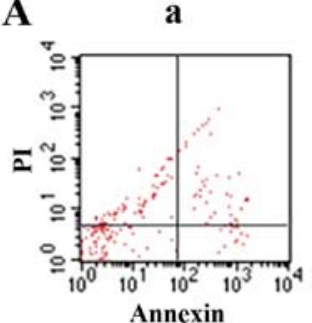

B

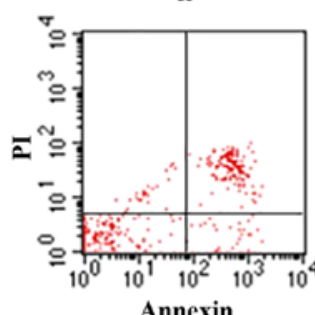

b

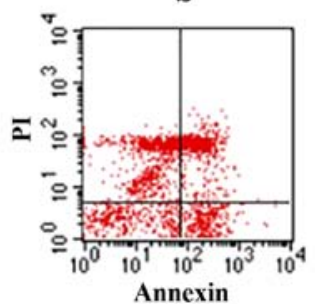

b

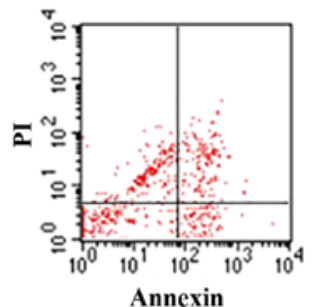

c

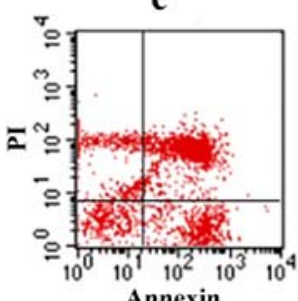

c

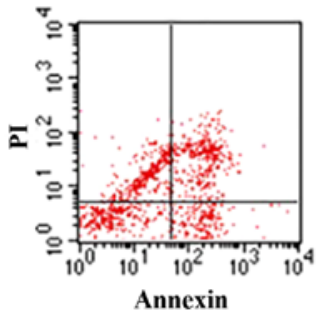

d

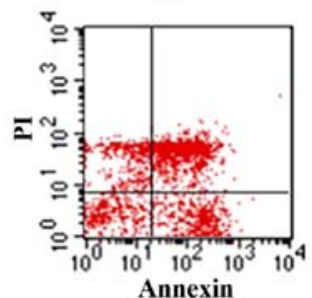

d

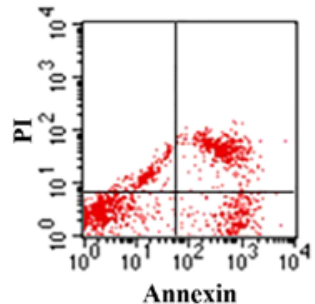

Figure 2. Cell apoptosis detection by flow cytometry. ccRCC cells were stained with Annexin V/FITC and analyzed by flow cytometry, the percentage of apoptotic cells was gradually increased, $24 \mathrm{~h}$ after treatment with TMP. (A) 786-O; (B) A498. a, Vehicle; b, $25 \mu \mathrm{M} ; \mathrm{c}, 50 \mu \mathrm{M} ; \mathrm{d}, 100 \mu \mathrm{M}$.
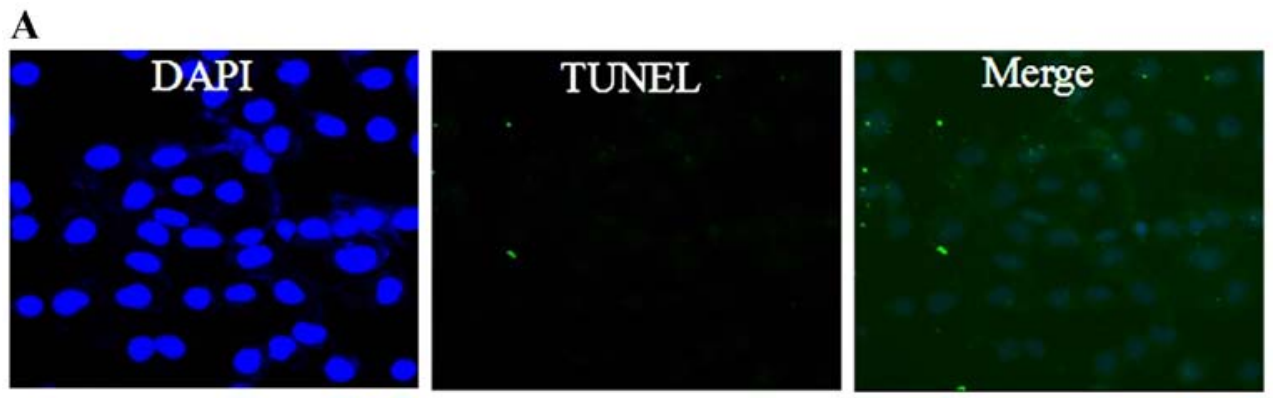

\section{B}
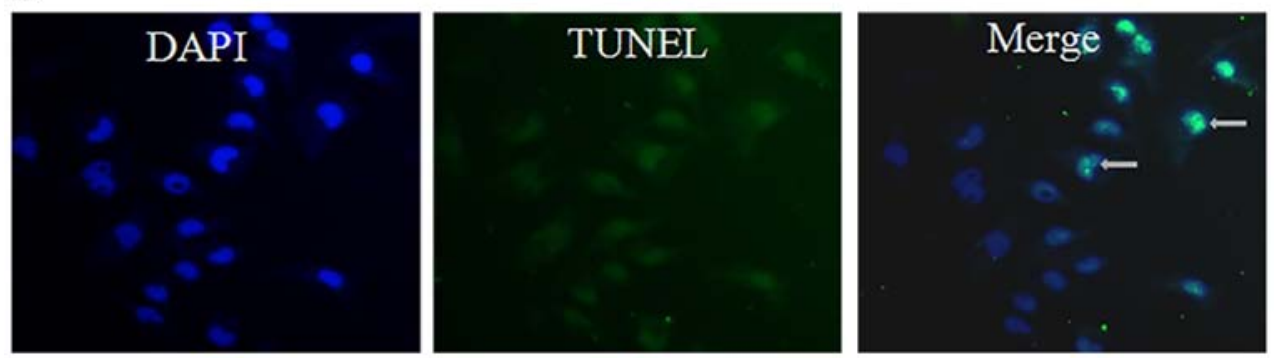

Figure 3. Cell apoptosis detection by TUNEL staining. 786-O cells were cultured with $100 \mu \mathrm{M}$ of TMP for $24 \mathrm{~h}$ an then stained with TUNEL (magnification, x200). (A) Vehicle; (B) TMP.

western blotting were used to assess EMT-related proteins (E-cadherin, vimentin and fibronectin) in 786-O cells. As shown in Figs. 6 and 7, the protein expression level of E-cadherin was significantly upregulated, but vimentin and fibronectin were significantly downregulation when the cells were treatment with $100 \mu \mathrm{M}$ of TMP for $24 \mathrm{~h}$. These results revealed that TMP was effective in inhibiting EMT process. Furthermore, western blotting results showed that the protein expression of MMP-9 and TGF- $\beta 1$ were significantly decreased after treatment with $100 \mu \mathrm{M}$ TMP for $24 \mathrm{~h}$. These results indicated the collapse of MMP and the inhibition of TGF- $\beta 1$ signaling in ccRCC cells when treated with TMP.
TMP upregulates the expression of MICA/MICB on the surface of ccRCC cell lines. In our previously report, the expression of MICA/B was significantly higher in 786-O than normal renal cells, but there was no different in A498 cells. To investigate the effect of TMP on the surface expression of NKG2D ligands in renal carcinoma cells, we initially performed a flow cytometric analysis and reverse transcription polymerase chain reaction (RT-PCR) analysis on 786-O and A498 cells. As shown in Fig. 8, 786-O and A498 cells showed apparent upregulation of MICA and MICB after treated with TMP, indicating that the inhibition of TMP on renal cancer cells may be associated with NKG2D-MICA signaling. 

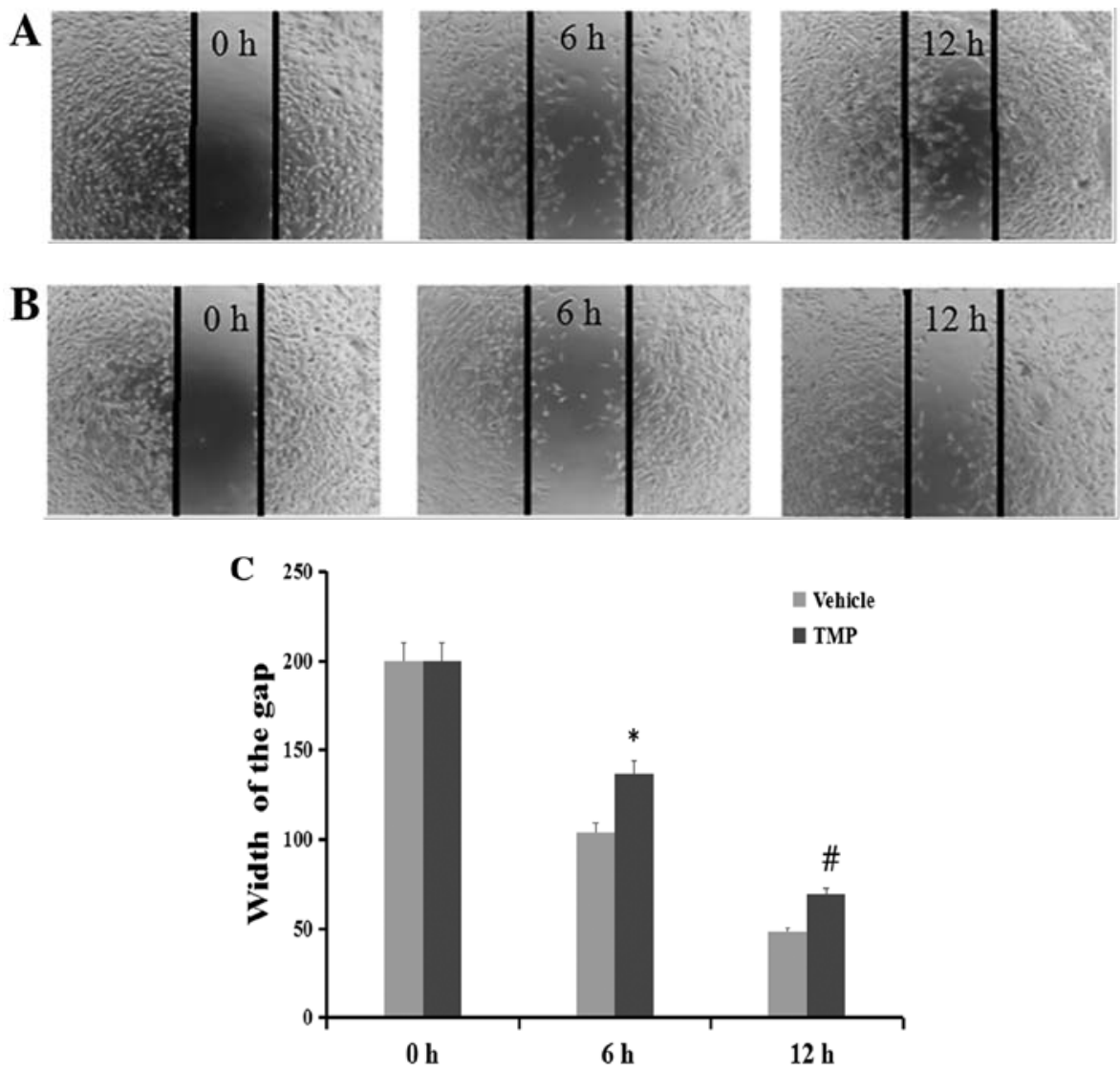

Figure 4. Wound healing analysis. TMP significantly inhibited the migratory ability of 786-O at the concentration of $100 \mu \mathrm{M}$ at 6 and $12 \mathrm{~h}$. (A) vehicle; (B) TMP; (C) comparison of the migratory ability.
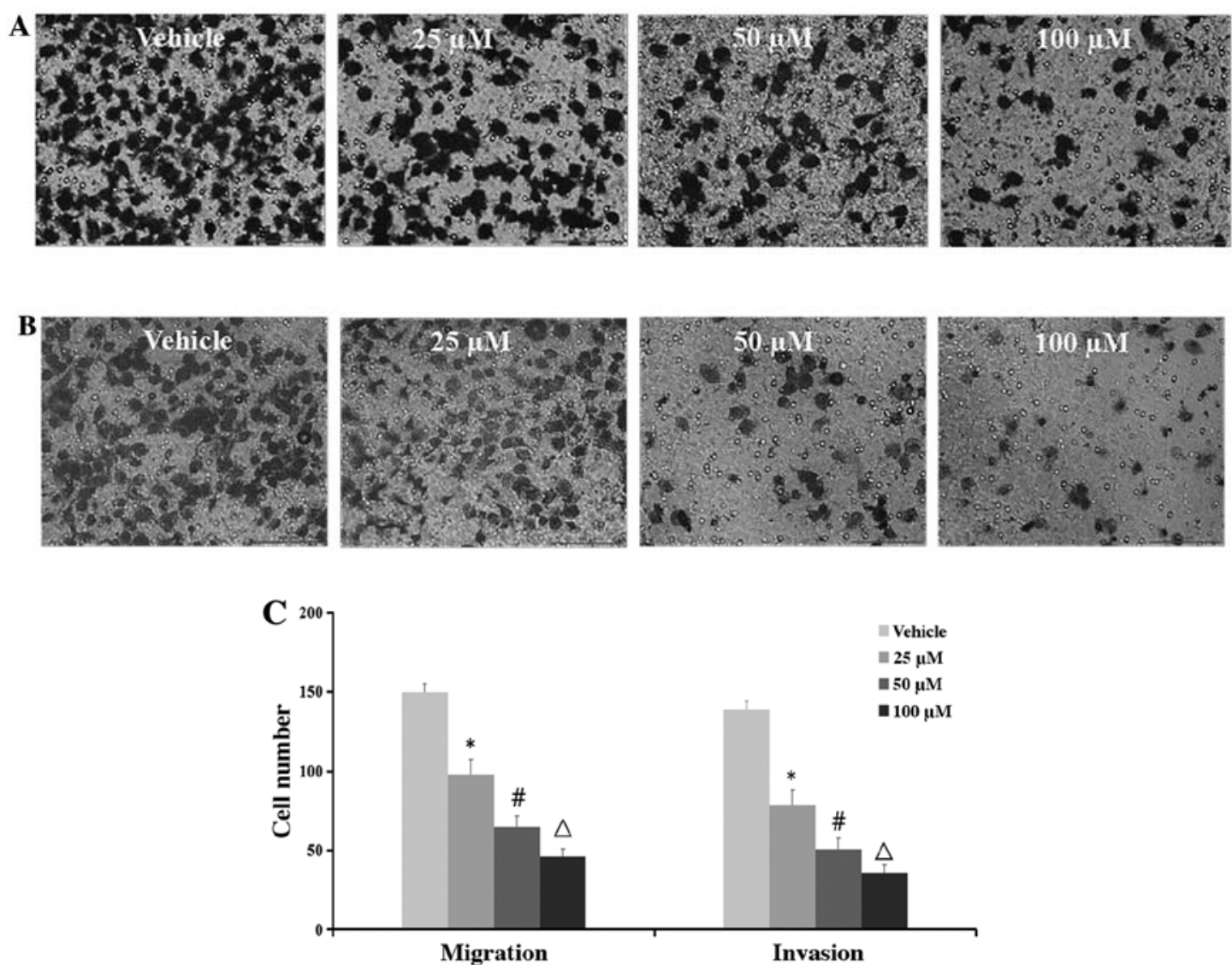

Figure 5. Cell migration and invasion analysis by Transwell assays. (A) Migration; (B) invasion; (C) comparison of the migration and invasion ability. 
A

a
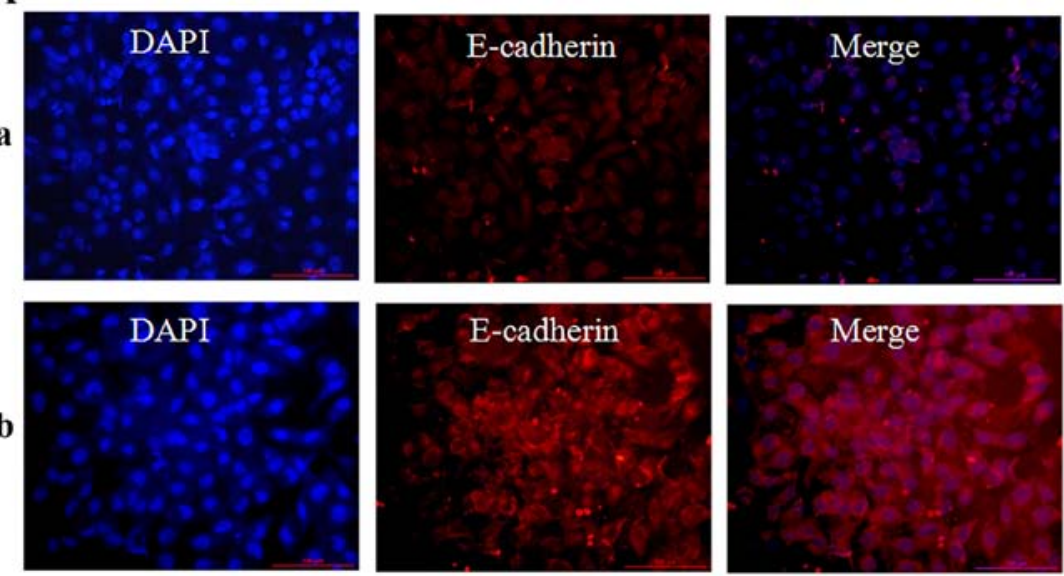

B
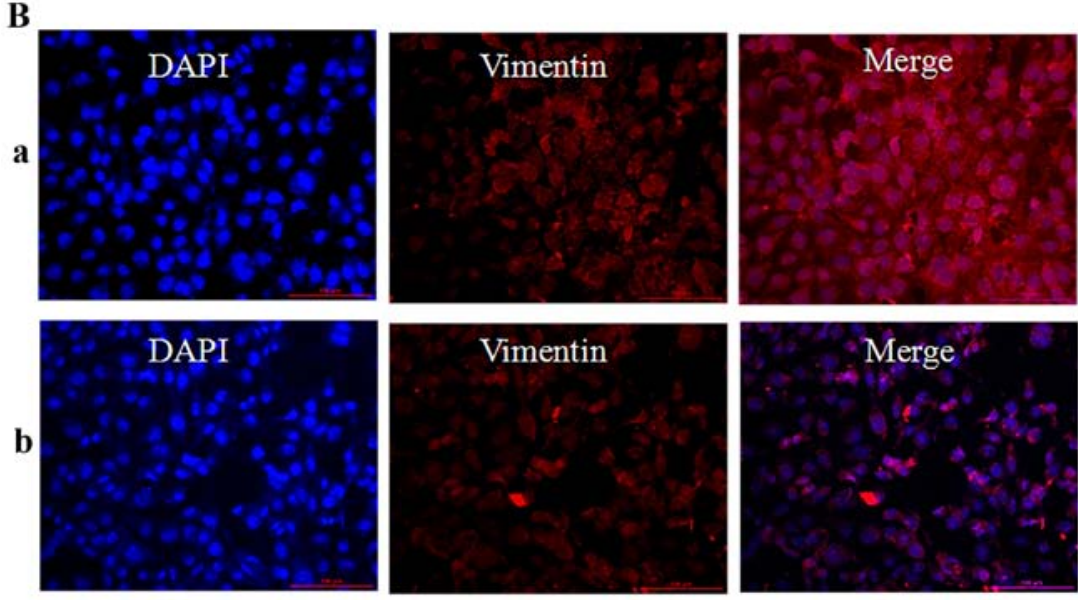

Figure 6. Epithelial-to-mesenchymal transition markers detection by immunofluorescence techniques. EMT-related proteins E-cadherin and vimentin in 786-O cells. a, vehicle; b, TMP.

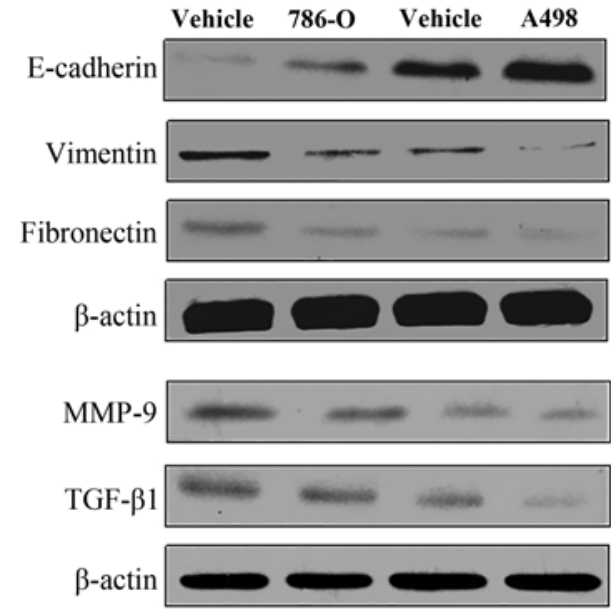

Figure 7. Western blotting detection. The protein expression level of EMT markers (E-cadherin, vimentin and fibronectin), MMP-9 and TGF- $\beta 1$ were analyzed when the cells were treated with $100 \mu \mathrm{M}$ of TMP for $24 \mathrm{~h}$.

TMP enhances susceptibility of ccRCC cells to NK cellmediated cytotoxicity. In order to detect the correlation of NKG2D signaling pathway, the interactions were blocked with saturating amounts of anti-MICA/B mAb. The effect of TMP on ccRCC cells to NK cell-mediated cytotoxicity was detected.
Two renal carcinoma cell lines were cultured for $24 \mathrm{~h}$ in the presence of $100 \mu \mathrm{M}$ TMP and the susceptibility to cytotoxicity of NK cells was examined. As shown in Fig. 9, TMP significantly increased the susceptibility of ccRCC cells to NK cells. Furthermore, NK cells pretreatment with MICA/B blocking antibody could partially prevent the cytotoxic activity of NK cells against all target cells treated with $100 \mu \mathrm{M}$ TMP.

\section{Discussion}

The present study demonstrated that TMP inhibited growth and induction of apoptosis in clear cell renal cell carcinoma (ccRCC) cell lines in a concentration- and time-dependent manner. Based on the results, the invasion and migration were significantly suppressed when treated with $100 \mu \mathrm{M}$ TMP through wound healing and Transwell assays in 786-O cells and A498 cells. Moreover, TMP suppressed the EMT pathway through downregulation the protein expression levels of epithelial marker E-cadherin and upregulation of mesenchymal markers vimentin and fibronectin in ccRCC cell lines 786-O and A498. Clear cell renal cell carcinoma is one of the most common kidney cancers, and increasing number of studies have demonstrated that epithelial-mesenchymal transition is associated with renal cell carcinoma invasion and metastasis (25-27). Therefore, the anticancer effect of TMP on 
$\mathbf{A}$
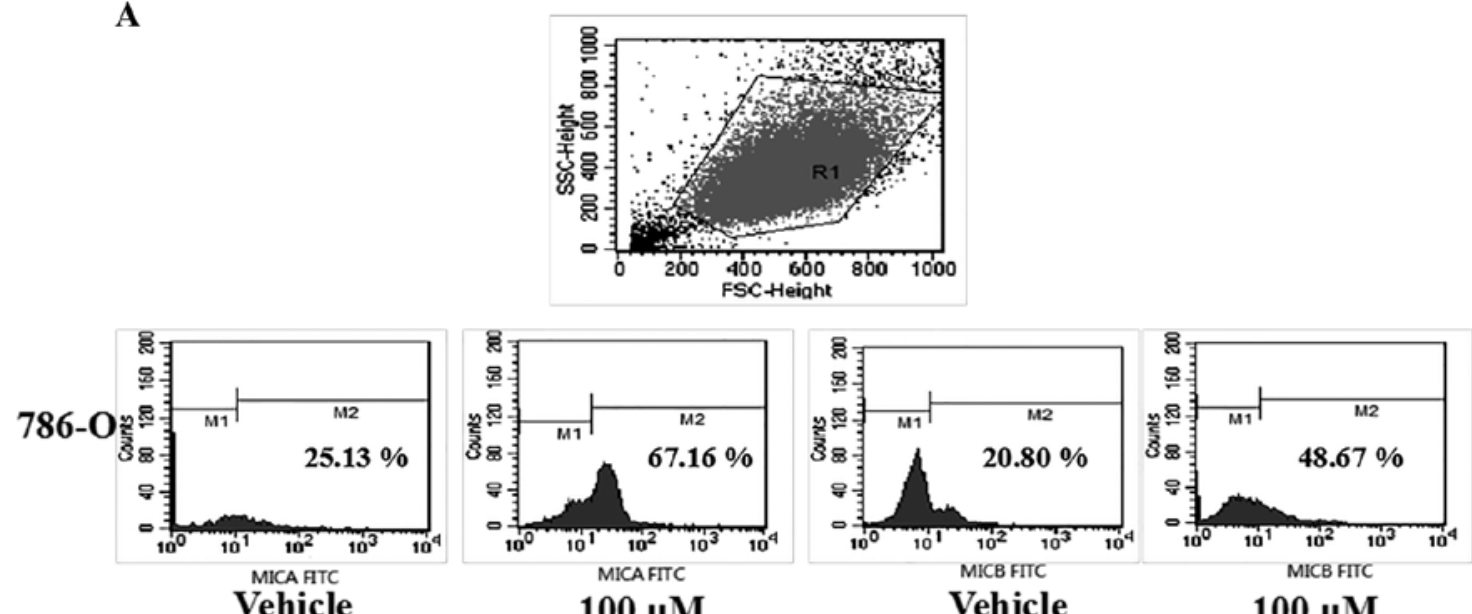

$100 \mu \mathrm{M}$
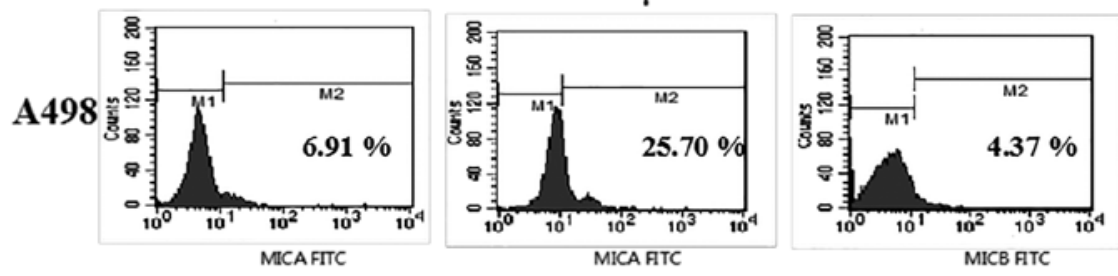

$100 \mu \mathrm{M}$
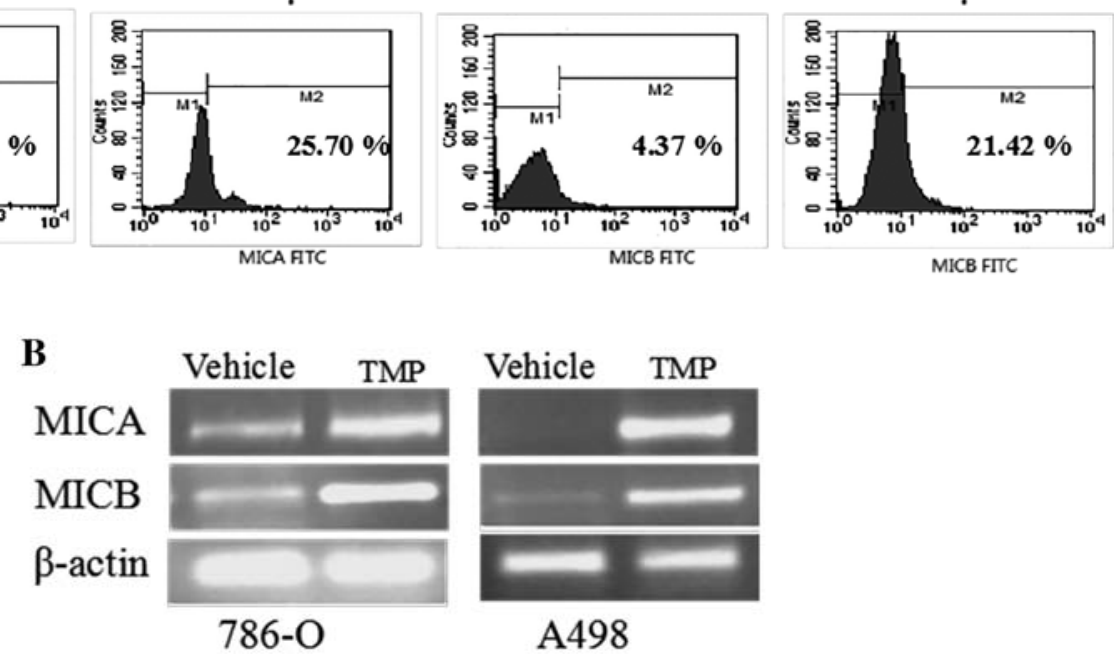

Figure 8. Expression of MICA and MICB on the surface of 786-O and A498 cells. (A) Flow cytometric analysis. (B) Reverse transcription polymerase chain reaction (RT-PCR) analysis.

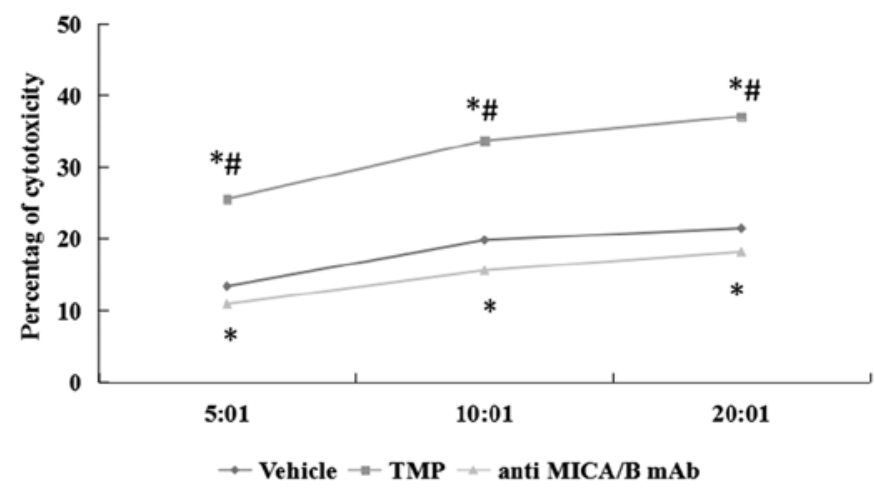

Figure 9. TMP enhances susceptibility of ccRCC cells to NK cell-mediated cytotoxicity. Two renal carcinoma cell lines were cultured for $24 \mathrm{~h}$ in the presence of $100 \mu \mathrm{M}$ TMP, and the susceptibility to cytotoxicity of NK cells was evaluated.

ccRCC cells might be mediated through suppressing the EMT pathway, thus supporting the potential of TMP to be developed as a promising agent for treatment of cancer. In agreement with previous studies, our findings contribute to the studies on biomarkers of kidney cancer and the mechanism of renal cancer progression driven by EMT pathway. Although there have been several studies on the molecular regulation of EMT, the mechanism is still not clear.

Major histocompatibility complex class I-related chains A and B (MICA/B), a ligand of Natural killer group 2, member $\mathrm{D}(\mathrm{NKG} 2 \mathrm{D})$ receptor, is broadly upregulated in epithelial originated tumor cells, which play a critical role in the immune surveillance against tumor cells and are associated with the prognosis of several malignancies (28). MICA/B is a ligand for the activating receptor NKG2D, the binding of NKG2DLs to NKG2D supports the cytotoxic effect of NK cells and T cells against tumor cells. Mounting evidence indicates that malignant epithelial cell polarization loss during the EMT allows the interaction of NKG2DL with NKG2D, leading to the activation of antitumor immune responses, which suggest that the correlation of NKG2D and NKG2DLs expression with the presence of EMT characteristics (20). However, the regulation of the NKG2D signaling during the EMT of carcinomas have yet to be elucidated. EMT is a morphogenetic process characterized by the acquisition of mesenchymal properties linked with an invasive phenotype and metastasis of tumor cells. Induction of EMT by TGF- $\beta$ stimulation strongly upregulated the expression of NKG2D ligands (NKG2DLs), MICA/B and ULBP1-3 in several epithelial tumor cell lines 
and by Snaill overexpression in colorectal cancer cells (29). Loss of epithelial integrity observed during the EMT process may be an important point of control of cancer progression by NKG2D-mediated immune mechanisms. Accordingly, with the increased expression of NKG2DLs, triggering of EMT rendered cancer cells more susceptible to NKG2D-mediated killing by NK cells $(23,30)$. Decrease of MICA/B expression was associated with a dramatic increase of NKG2D(+)-tumor infiltrating lymphocytes in vivo. In our previous study (21), we found that the content of soluble MICA (sMICA) in serum of patients with renal cell carcinoma was significantly higher than in healthy adults, the percentage of membrane MICA (mMICA) were significantly increased in human kidney cancer tissues and ccRCC cell line 786-O and Ketr-3, but no higher in A498 cells. Furthermore, we indicated that the relationship of expression of NKG2D and MICA/B play an important role in mediation of the cytotoxic NK cells in ccRCCs and sMICA mediated tumor immune escape through downregulated NKG2D expression. However, the underlying molecular mechanisms in the tumor microenvironment in ccRCC lesions is not yet clarified. In the present study, based on the results of PCR and FACS, the expression of MICA and MICB were higher in 786-O, but not A498, which is in agreement with our previous results. Interestingly, the antitumor effects of TMP were more significant in MICA high expression RCC cell lines 786-O than low expression RCC cell lines A498. Thus, we hypothesize that TMP inhibit proliferation, migration and invasion of ccRCC cells through regulation of NKG2D-NKGDLs signaling during the EMT. In order to further revealed the mechanism, the interactions were blocked with saturating amounts of anti-MICA/B mAb, the effect of TMP on ccRCC cells to NK cell-mediated cytotoxicity were detected and the results showed that TMP significantly increased the susceptibility of ccRCC cells to NK cells. EMT is primarily mediated by local production and activation of TGF- $\beta 1$, EMT is also a relevant checkpoint in the control of tumor progression through NKG2D-mediated immune responses. Many studies have been conducted on the relationship between matrix metalloproteinases (MMPs) expression in cancer patients. MMP-9 due to their proteolytic nature degrade proteins that regulate various cellular behaviors related to cancer cell differentiation, migration, invasion, and surveillance of the immune system (31). In our results, treatment with TMP, the protein levels of TGF- $\beta 1$ and MMP-9 were lower in ccRCC cells indicating that the mechanism of suppression EMT may be related through TGF- $\beta 1$ and MMP signal pathway. Previous studies reported that TGF- $\beta$ downregulates the expression of NKG2D and its ligands in different tumor types. Taken together, the antitumor effect of TMP is a complicated process, in which the NKG2D-NKG2DL signaling plays a key role.

In summary, our results suggested that TMP possess the activity of anti-proliferation and apoptosis induction in human clear cell renal cell carcinoma (ccRCC) cell lines, 786-O and A498 cells. Interestingly, the inhibition was more significantly increased in 786-O than in A498 cells. Our following studies indicated that the molecular mechanisms may be mediated through inhibition of NKG2D related signaling pathway to further suppress EMT progression. The binding of NKG2D to its ligands activates NK cells, which gives the rationale for studies on the utilization of TMP as a potential cancer thera- peutic compound to increase NK cell-mediated cytotoxicity against high NKG2DL expressing cancer cells.

\section{Acknowledgements}

We are grateful to Central Research Laboratory, the Second Hospital of Shandong University for technical assistance and the generous support. The present study was supported by a grant from the National Natural Science Foundation of China (grant no. 81500042), the Natural Science Foundation of Shandong Province (grant nos. ZR2014HQ045 and ZR2013HM103) and the Medical and Health Science Technology Development Plan Project of Shandong Province (grant no. 2015WS0307).

\section{References}

1. Yan Y, Zhao J, Cao C, Jia Z, Zhou N, Han S, Wang Y, Xu Y, Zhao J, Yan Y, et al: Tetramethylpyrazine promotes SH-SY5Y cell differentiation into neurons through epigenetic regulation of Topoisomerase II $\beta$. Neuroscience 278: 179-193, 2014.

2. Wang L, Zhang X, Cui G, Chan JY, Wang L, Li C, Shan L, Xu C, Zhang Q, Wang Y, et al: A novel agent exerts antitumor activity in breast cancer cells by targeting mitochondrial complex II. Oncotarget: Mar 27, 2016 (Epub ahead of print).

3. Kao TK, Chang CY, Ou YC, Chen WY, Kuan YH, Pan HC, Liao SL, Li GZ and Chen CJ: Tetramethylpyrazine reduces cellular inflammatory response following permanent focal cerebral ischemia in rats. Exp Neurol 247: 188-201, 2013.

4. Liu X, Liu H, Zeng Z, Zhou W, Liu J and He Z: Pharmacokinetics of ligustrazine ethosome patch in rats and anti-myocardial ischemia and anti-ischemic reperfusion injury effect. Int $\mathrm{J}$ Nanomed 6: 1391-1398, 2011

5. Jia Y, Wang Z, Zang A, Jiao S, Chen S and Fu Y: Tetramethylpyrazine inhibits tumor growth of lung cancer through disrupting angiogenesis via BMP/Smad/Id-1 signaling. Int J Oncol 48: 2079-2086, 2016

6. Kim M, Kim SO, Lee M, Lee JH, Jung WS, Moon SK, Kim YS, Cho KH, Ko CN and Lee EH: Tetramethylpyrazine, a natural alkaloid, attenuates pro-inflammatory mediators induced by amyloid $\beta$ and interferon- $\gamma$ in rat brain microglia. Eur J Pharmacol 740: 504-511, 2014.

7. Wang XJ, Xu YH, Yang GC, Chen HX and Zhang P: Tetramethylpyrazine inhibits the proliferation of acute lymphocytic leukemia cell lines via decrease in GSK-3 3 . Oncol Rep 33: 2368-2374, 2015.

8. Zhang P, Zheng BB, Wang HY, Chen JH, Liu XY and Guo XL: DLJ14, a novel chemo-sensitization agent, enhances therapeutic effects of adriamycin against MCF-7/A cells both in vitro and in vivo. J Pharm Pharmacol 66: 398-407, 2014.

9. Yi B, Liu D, He M, Li Q, Liu T and Shao J: Role of the ROS/ AMPK signaling pathway in tetramethylpyrazine-induced apoptosis in gastric cancer cells. Oncol Lett 6: 583-589, 2013.

10. Chen Z, Pan X, Georgakilas AG, Chen P, Hu H, Yang Y, Tian S, Xia L, Zhang J, Cai X, et al: Tetramethylpyrazine (TMP) protects cerebral neurocytes and inhibits glioma by down regulating chemokine receptor CXCR4 expression. Cancer Lett 336: 281-289, 2013.

11. Zheng CY, Xiao W, Zhu MX, Pan XJ, Yang ZH and Zhou SY: Inhibition of cyclooxygenase-2 by tetramethylpyrazine and its effects on A549 cell invasion and metastasis. Int J Oncol 40: 2029-2037, 2012

12. Pan H, Jiang T, Cheng N, Wang Q, Ren S, Li X, Zhao C, Zhang L, Cai W and Zhou C: Long non-coding RNABC087858 induces nonT790M mutation acquired resistance to EGFR-TKIs by activating PI3K/AKT and MEK/ERK pathways and EMT in non-small-cell lung cancer. Oncotarget: Jul 9, 2016 (Epub ahead of print).

13. Bonyadi Rad E, Hammerlindl H, Wels C, Popper U, Ravindran Menon D, Breiteneder H, Kitzwoegerer M, Hafner C, Herlyn M, Bergler H, et al: Notch4 signaling induces a mesenchymal-epithelial-like transition in melanoma cells to suppress malignant behaviors. Cancer Res 76: 1690-1697, 2016.

14. Raza U, Saatci Ö, Uhlmann S, Ansari SA, Eyüpoğlu E, Yurdusev E, Mutlu M, Ersan PG, Altundağ MK and Zhang J: The miR-644a/ CTBP1/p53 axis suppresses drug resistance by simultaneous inhibition of cell survival and epithelial-mesenchymal transition in breast cancer. Oncotarget: Jul 8, 2016 (Epub ahead of print). 
15. Matusali G, Tchidjou HK, Pontrelli G, Bernardi S, D' Ettorre G Vullo V, Buonomini AR, Andreoni M, Santoni A, Cerboni C and Doria M: Soluble ligands for the NKG2D receptor are released during HIV-1 infection and impair NKG2D expression and cytotoxicity of NK cells. FASEB J 27: 2440-2450, 2013.

16. Guerra N, Tan YX, Joncker NT, Choy A, Gallardo F, Xiong N, Knoblaugh S, Cado D, Greenberg NM and Raulet DH: NKG2Ddeficient mice are defective in tumor surveillance in models of spontaneous malignancy. Immunity 28: 571-580, 2008.

17. López-Larrea C, Suárez-Alvarez B, López-Soto A, LópezVázquez A and Gonzalez S: The NKG2D receptor: Sensing stressed cells. Trends Mol Med 14: 179-189, 2008.

18. Cosman D, Müllberg J, Sutherland CL, Chin W, Armitage R, Fanslow W, Kubin M and Chalupny NJ: ULBPs, novel MHC class I-related molecules, bind to CMV glycoprotein UL16 and stimulate NK cytotoxicity through the NKG2D receptor. Immunity 14: 123-133, 2001.

19. López-Soto A, Zapico LH, Acebes-Huerta A, Rodrigo L and Gonzalez S: Regulation of NKG2D signaling during the epithelial-to-mesenchymal transition. Oncoimmunology 2: e25820, 2013.

20. Ni D, Ma X, Li HZ, Gao Y, Li XT, Zhang Y, Ai Q, Zhang P, Song EL, Huang QB, et al: Downregulation of FOXO3a promotes tumor metastasis and is associated with metastasis-free survival of patients with clear cell renal cell carcinoma. Clin Cancer Res 20: 1779-1790, 2014.

21. Jia HY, Liu JL, Yuan MZ, Zhou CJ, Sun WD, Zhao JJ, Wang J, Liu L and Luan Y: Regulation roles of MICA and NKG2D in human renal cancer cells. Asian Pac J Cancer Prev 16: 3901-3905, 2015.

22. Subramani R, Gonzalez E, Nandy SB, Arumugam A, Camacho F, Medel J, Alabi D and Lakshmanaswamy R: Gedunin inhibits pancreatic cancer by altering sonic hedgehog signaling pathway. Oncotarget: Mar 14, 2016 doi: 10.18632/oncotarget.8055 (Epub ahead of print).

23. Friese MA, Wischhusen J, Wick W, Weiler M, Eisele G, Steinle A and Weller M: RNA interference targeting transforming growth factor-beta enhances NKG2D-mediated antiglioma immune response, inhibits glioma cell migration and invasiveness, and abrogates tumorigenicity in vivo. Cancer Res 64: 7596-7603, 2004.
24. Yang F, Shao Y, Yang F, Liu M, Huang J, Zhu K, Guo C, Luo J, Li W, Yang B, et al: Valproic acid upregulates NKG2D ligand expression and enhances susceptibility of human renal carcinoma cells to NK cell-mediated cytotoxicity. Arch Med Sci 9: 323-331, 2013.

25. Mikami S, Mizuno R, Kosaka T, Saya H, Oya M and Okada Y: Expression of TNF- $\alpha$ and CD44 is implicated in poor prognosis, cancer cell invasion, metastasis and resistance to the sunitinib treatment in clear cell renal cell carcinomas. Int J Cancer 136: 1504-1514, 2015.

26. Hill B, De Melo J, Yan J, Kapoor A, He L, Cutz JC, Feng X, Bakhtyar $\mathrm{N}$ and Tang D: Common reduction of the Raf kinase inhibitory protein in clear cell renal cell carcinoma. Oncotarget 5: 7406-7419, 2014

27. Lawrie CH, Larrea E, Larrinaga G, Goicoechea I, Arestin M, Fernandez-Mercado M, Hes O, Cáceres F, Manterola L and López JI: Targeted next-generation sequencing and non-coding RNA expression analysis of clear cell papillary renal cell carcinoma suggests distinct pathological mechanisms from other renal tumour subtypes. J Pathol 232: 32-42, 2014.

28. Zhang X, Yan L, Jiao W, Ren J, Xing N, Zhang Y, Zang Y, Wang $\mathrm{J}$ and $\mathrm{Xu} Z \mathrm{Z}$ : The clinical and biological significance of MICA in clear cell renal cell carcinoma patients. Tumour Biol 37: 2153-2159, 2016.

29. López-Soto A, Huergo-Zapico L, Galván JA, Rodrigo L, de Herreros AG, Astudillo A and Gonzalez S: Epithelialmesenchymal transition induces an antitumor immune response mediated by NKG2D receptor. J Immunol 190: 4408-4419, 2013.

30. Eisele G, Wischhusen J, Mittelbronn M, Meyermann R, Waldhauer I, Steinle A, Weller M and Friese MA: TGF- $\beta$ and metalloproteinases differentially suppress NKG2D ligand surface expression on malignant glioma cells. Brain 129: 2416-2425, 2006

31. Kunigal S, Lakka SS, Joseph P, Estes N and Rao JS: Matrix metalloproteinase-9 inhibition down-regulates radiation-induced nuclear factor-kappa B activity leading to apoptosis in breast tumors. Clin Cancer Res 14: 3617-3626, 2008 